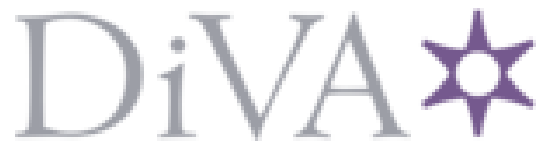

http://www.diva-portal.org

Preprint

This is the submitted version of a paper presented at IEEE Information Theory Workshop (ITW), NOV 25-29, 2018, Guangzhou, PEOPLES R CHINA.

Citation for the original published paper:

Vu, M T., Oechtering, T J., Skoglund, M. (2018)

Testing in Identification Systems

In: 2018 IEEE INFORMATION THEORY WORKSHOP (ITW) (pp. 295-299). IEEE

Information Theory Workshop

https://doi.org/10.1109/ITW.2018.8613310

N.B. When citing this work, cite the original published paper.

The work was supported by the Swedish Research Council (VR) project under Grant 2016-03853.

Permanent link to this version:

http://urn.kb.se/resolve?urn=urn:nbn:se:kth:diva-252670 


\title{
Testing in Identification Systems
}

\author{
Minh Thanh Vu, Tobias J. Oechtering and Mikael Skoglund \\ Department of Information Science and Engineering \\ KTH-Royal Institute of Technology
}

\begin{abstract}
We study a hypothesis testing problem to decide whether or not an observation sequence is related to one of users in a database which contains compressed versions of users' data. Our main interest lies on the characterization of the exponent of the probability of the second kind of error when the number of users in the database grows exponentially. We show a lower bound on the error exponent and identify special cases where the bound is tight. Next, we study the $\epsilon$-achievable error exponent and show a sub-region where the lower bound is tight.
\end{abstract}

Index Terms-Mixture distribution, information-spectrum method, strong converse.

\section{INTRODUCTION}

We consider the following setting in this work. Assume that there is a database in the system which stores compressed versions of data sequences of $M$ users $\left(x^{n}(m)\right)_{m=1}^{M}$. A observation sequence $y^{n}$ is provided to a processing center which has access to the compressed data sequences. The processing center performs a screening step and returns Yes/No when $y^{n}$ is related to one of the user/ independent of all users in the system. We call the first case hypothesis $H_{0}$ and the second case hypothesis $H_{1}$.

Our problem is motivated by the identification system investigated in [1] where the task is to identify the correct user based on the observation $y^{n}$ and the information inside the database $\left(x^{n}(i)\right)_{i=1}^{M}$. Therein $y^{n}$ is an output of an observation channel $P_{Y \mid X}$ where the input is selected uniformly at random from the database $\left(x^{n}(i)\right)_{i=1}^{M}$. The compression of users' data is considered in [2], [3]. In contrast, we are not interested in identifying the true user even if the sequence $y^{n}$ is indeed related to one user in the system. Instead, we only want to know if the sequence is related to the database.

Other hypothesis testing problems related to the identification problem include [4]-[6]. In [4] the hypothesis $H_{1}$ was tested against $M$ other hypotheses in the binary setting where the focus was to minimize the overall identification error under a specific decision rule. In [5] the author considered the $M$-ary hypothesis testing problem with fixed $M$ and studied the large deviation regime. In [6] the decision rule was based on a decoding metric using the hashed data and observation sequences at different lengths. The exponents of the probability of miss and the expected number of incorrect items on the list were provided for a fixed hashed function.

In this work we first study the exponent of the probability of the second kind of error $E^{\star}\left(R, R_{c}\right)$ provided that the probability of the first kind is vanishing. $E^{\star}\left(R, R_{c}\right)$ depends on $R_{c}$, the compression rate, and $R$, the identification rate in the context of [1], which represents the number of stored sequences in the database. We provide a lower bound on $E^{\star}\left(R, R_{c}\right)$ when $R<R_{\max }\left(R_{c}\right)$ by the random coding method where $R_{\max }\left(R_{c}\right)$ is the identification capacity given the compression rate $R_{c}$ studied in [2]. It is then shown that the lower bound is tight in the weak converse sense in special cases: lossless compression, sub-exponential number of users. Additionally, we show that the lower bound is tight in the strong converse sense if $R$ is less than half of the threshold value $R_{\max }\left(R_{c}\right)$.

The case of $M=1$ is investigated by Ahlswede and Csiszar in [7] where both weak and strong converses are shown. Due to the non-stationary of our setting, we employ informationspectrum techniques to derive the results.

\section{A LOWER BOUnd OF $E^{\star}\left(R, R_{c}\right)$}

We assume that the database consists of $M$ users where ${ }^{1}$ $M \doteq e^{n R}$, i.e., $\lim _{n \rightarrow \infty} \frac{1}{n} \log M=R$. We consider in this work only the discrete scenario where $\mathcal{X}$ and $\mathcal{Y}$ are finite. For each $i$ the corresponding data sequence $x^{n}(i)$ is generated iid from the distribution $P_{X}$. The data sequences are mutually independent. Under $H_{0}$ the joint distribution of the sequence $y^{n}$ and sequences $\left(x^{n}(m)\right)_{m=1}^{M}$ is given by ${ }^{2}$

$$
P_{H_{0}}=\sum_{i=1}^{M} \frac{1}{M} P_{Y \mid X}^{\otimes n} P_{X^{n}(i)} \times \prod_{k=1, k \neq i}^{M} P_{X^{n}(k)},
$$

i.e., the sequence $y^{n}$ is related to one randomly chosen user in the system. The joint distribution under $H_{1}$ is given by

$$
P_{H_{1}}=P_{Y}^{\otimes n} \times \prod_{i=1}^{M} P_{X^{n}(i)},
$$

i.e., the sequence $y^{n}$ is not related to the information in the database. We note that under both hypotheses the joint distributions are non-iid as $M$ increases with the block length $n$.

Definition 1. A testing scheme consists of a compression mapping which enrolls the users' data sequences into the database according to

$$
\phi_{n}: \mathcal{X}^{n} \rightarrow \mathcal{M}_{1}
$$

and a decision mapping which outputs whether $H_{0}$ or $H_{1}$ is deemed true

$$
\psi_{n}: \mathcal{Y}^{n} \times \mathcal{M}_{1}^{M} \rightarrow\{0,1\}
$$

\footnotetext{
${ }^{1} \log$ is taken to the natural base.

${ }^{2}$ For a measure $\mu, \mu^{\otimes n}$ denotes its $n$-fold product extension.
} 
The acceptance region is defined accordingly as

$$
\mathcal{A}_{n}=\left\{\left(y^{n},\left(x^{n}(i)\right)_{i=1}^{M}\right) \mid \psi_{n}\left(y^{n},\left(j_{i}\right)_{i=1}^{M}\right)=0\right\},
$$

where $j_{i}=\phi_{n}\left(x^{n}(i)\right)$, for all $i \in[1: M]$. The probability of first and second type of error are given respectively as

$$
\alpha_{n}=P_{H_{0}}\left(\mathcal{A}_{n}^{c}\right), \beta_{n}=P_{H_{1}}\left(\mathcal{A}_{n}\right) .
$$

Definition 2. An error exponent $E$ of type II is achievable given $\left(R, R_{c}\right)$ if there exist enrollment and decision mappings $\left(\phi_{n}, \psi_{n}\right)$ such that

$$
\begin{gathered}
\lim _{n \rightarrow \infty} \alpha_{n}=0, \limsup _{n \rightarrow \infty} \frac{1}{n} \log \left|\mathcal{M}_{1}\right| \leq R_{c}, \\
\liminf _{n \rightarrow \infty} \frac{1}{n} \log \frac{1}{\beta_{n}} \geq E .
\end{gathered}
$$

We define

$$
E^{\star}\left(R, R_{c}\right)=\sup \left\{E \mid E \text { is achievable given }\left(R, R_{c}\right)\right\} .
$$

Remark 1. An easy observation when the compression is at zero-rate, i.e., the compression alphabet is of sub-exponential size, is

$$
E^{\star}(R, 0)=0, \forall R .
$$

To interpret the result we look at $I\left(Y^{n} ;\left(J_{i}\right)_{i=1}^{M}\right)$ under $H_{0}$ where $J_{i}=\phi_{n}\left(X^{n}(i)\right), \forall i \in[1: M]$. Let $W$ be the hidden random variable that characterizes the uniformly chosen user in the hypothesis $H_{0}$ which is independent of users' sequences, i.e.,

$$
\begin{aligned}
& P_{H_{0}}\left(y^{n},\left(x^{n}(i)\right)_{i=1}^{M}, W=w\right) \\
& \quad=\frac{1}{M} P_{Y X}^{\otimes n}\left(y^{n}, x^{n}(w)\right) \prod_{i \neq w} P_{X}^{\otimes n}\left(x^{n}(i)\right) .
\end{aligned}
$$

Note that

$$
\begin{aligned}
I_{H_{0}}\left(Y^{n} ;\left(J_{i}\right)_{i=1}^{M}\right) & \leq I_{H_{0}}\left(Y^{n} ; W, J_{W}\right) \\
& =I_{H_{0}}\left(Y^{n} ; J_{W} \mid W\right) \leq \log \left|\mathcal{M}_{1}\right| .
\end{aligned}
$$

When the compression is done at zero-rate then we have that $n H(Y) \approx n\left(H(Y)-1 / n \log \left|\mathcal{M}_{1}\right|\right) \leq H\left(Y^{n} \mid\left(J_{i}\right)_{i=1}^{M}\right) \leq$ $n H(Y)$ since $1 / n \log \left|\mathcal{M}_{1}\right|$ tends to 0 as $n \rightarrow \infty$. This implies roughly that asymptotically $Y^{n}$ is independent of $\left(J_{i}\right)_{i=1}^{M}$. As the marginal distributions of $Y^{n}$ and $\left(J_{i}\right)_{i=1}^{M}$ in both hypotheses are identical, the chance that we can distinguish them from each other is low asymptotically. Since the probability of the first kind of error $\alpha_{n} \rightarrow 0$, the probability of the second kind of error is bounded away from 0 due to the indistinguishability.

Given a compression rate $R_{c}$ we define the following functions

$$
\begin{aligned}
& R_{\max }\left(R_{c}\right)=\max _{\substack{U-X-Y,|\mathcal{U}| \leq|\mathcal{X}|+1, I(X ; U) \leq R_{c}}} I(Y ; U) \\
& \theta\left(R, R_{c}\right)=R_{\max }\left(R_{c}\right)-R \text {, on } 0 \leq R<R_{\max }\left(R_{c}\right) .
\end{aligned}
$$

We have the following lower bound on $E^{\star}\left(R, R_{c}\right)$.
Theorem 1. Assume that the condition $0 \leq R<R_{\max }\left(R_{c}\right)$ is satisfied, then

$$
E^{\star}\left(R, R_{c}\right) \geq \theta\left(R, R_{c}\right) .
$$

It is difficult to apply the same trick using Stein's lemma as in the proof of [7, Theorem 1] mainly as the number of components $M$ in both distributions grows with $n$. For brevity, in the sequel we abbreviate $R_{\max }\left(R_{c}\right)$ as $R_{\max }$. We use a random coding argument to show the lower bound.

Proof. Since $R<R_{\max }$ there exists for any $\delta>0$ a conditional distribution $P_{U \mid X}$ such that $R_{c}>I(X ; U)$ and $\max \left\{R, R_{\max }-\delta\right\}<I(Y ; U)<R_{\max }$ hold. We are going to show that $I(Y ; U)-R$ is an achievable exponent of the second type of error. Generate $e^{n R_{c}}$ codewords $u^{n}(m)$ iid according to the marginal distribution $P_{U}$.

Enrollment: For each user $i$, we look for a codeword $u^{n}\left(m_{i}\right)$ such that $\left(x^{n}(i), u^{n}\left(m_{i}\right)\right) \in \mathcal{T}_{\epsilon}^{n}\left(P_{X U}\right)$, where $\mathcal{T}_{\epsilon}^{n}$ denotes the strongly typical set, and store $m_{i}$ as $j_{i}$ into the database.

Acceptance region: $\mathcal{A}_{n}$ is defined (for a given codebook) as the set

$$
\begin{gathered}
\mathcal{A}_{n}=\left\{\left(y^{n},\left(x^{n}(i)\right)_{i=1}^{M}\right) \mid\left(y^{n}, u^{n}\left(j_{i}\right)\right) \in \mathcal{T}_{\epsilon}^{n}\left(P_{Y U}\right)\right. \\
\text { for some } i\} .
\end{gathered}
$$

Analysis: We analyze the probabilities of errors averaging over codebooks. Let $\left(U^{n}\left(J_{i}\right)\right)_{i=1}^{M}$ be the induced random variables by the enrollment process under $H_{0}$. Since

$$
\begin{aligned}
\alpha_{n} & =\operatorname{Pr}_{H_{0}}\left\{\mathcal{A}_{n}^{c}\right\}=\operatorname{Pr}_{H_{0}}\left\{\left(Y^{n}, U^{n}\left(J_{i}\right)\right) \notin \mathcal{T}_{\epsilon}^{n}\left(P_{Y U}\right), \forall i\right\} \\
& =\sum_{w} \operatorname{Pr}_{H_{0}}\left\{\left(Y^{n}, U^{n}\left(J_{i}\right)\right) \notin \mathcal{T}_{\epsilon}^{n}\left(P_{Y U}\right), \forall i, W=w\right\} \\
& \leq \sum_{w} \operatorname{Pr}_{H_{0}}\left\{\left(Y^{n}, U^{n}\left(J_{w}\right)\right) \notin \mathcal{T}_{\epsilon}^{n}\left(P_{Y U}\right), W=w\right\} \\
& =\operatorname{Pr}_{H_{0}}\left\{\left(Y^{n}, U^{n}\left(J_{W}\right)\right) \notin \mathcal{T}_{\epsilon}^{n}\left(P_{Y U}\right)\right\},
\end{aligned}
$$

where $\operatorname{Pr}_{H_{0}}\{\mathcal{E}\}$ means that the probability of the event $\mathcal{E}$ is evaluated using the distribution $P_{H_{0}}$. From the covering lemma as $R_{c}>I(X ; U)$ and the conditionally typical lemma [8] we have

$$
\alpha_{n} \rightarrow 0 \text {, as } n \rightarrow \infty \text {. }
$$

For notational clarity, let $\left(\bar{Y}^{n},(\bar{X}(i))_{i=1}^{M}\right)$ be the collection of random sequences distributed as $P_{H_{1}}$. Similarly, for each $i$ the induced "stored" sequence is denoted by $\bar{U}^{n}\left(\bar{J}_{i}\right)$. The probability of the second type of error can be upper bounded as

$$
\begin{aligned}
\beta_{n} & =\operatorname{Pr}_{H_{1}}\left\{\left(\bar{Y}^{n}, \bar{U}^{n}\left(\bar{J}_{i}\right)\right) \in \mathcal{T}_{\epsilon}^{n}\left(P_{Y U}\right) \text { for some } i\right\} \\
& \leq \sum_{i} \operatorname{Pr}_{H_{1}}\left\{\left(\bar{Y}^{n}, \bar{U}^{n}\left(\bar{J}_{i}\right)\right) \in \mathcal{T}_{\epsilon}^{n}\left(P_{Y U}\right)\right\} \\
& \leq M e^{-n\left(I(Y ; U)-\gamma_{n}\right)} \doteq e^{-n\left(I(Y ; U)-\gamma_{n}-R\right)},
\end{aligned}
$$

where $\gamma_{n} \rightarrow 0$ as $n \rightarrow \infty$. Therefore there exists a sequence of codebooks such that

$$
\lim _{n \rightarrow \infty} \alpha_{n}=0,
$$




$$
\liminf _{n \rightarrow \infty} \frac{1}{n} \log \frac{1}{\beta_{n}} \geq I(Y ; U)-R .
$$

Hence, this proves the theorem.

In the following, we investigate some cases where the lower bound is actually tight. For notation brevity we also abbreviate $\left(X^{n}(i)\right)_{i=1}^{M}$ as $\mathbf{X}^{n}$ and $\left(\phi_{n}\left(X^{n}(i)\right)\right)_{i=1}^{M}$ as $\phi_{n}\left(\mathbf{X}^{n}\right)$.

Proposition 1. Assume that the compression is lossless, then the lower bound is tight, i.e. the error exponent for the second type of error is given by

$$
E_{l l}^{\star}=I(Y ; X)-R, \text { for } R<I(Y ; X) .
$$

Proof. Denote the losslessly compressed sequences by $\left(\hat{X}_{i}^{n}\right)_{i=1}^{M}$ abbreviated in the following as $\hat{\mathbf{X}}^{n}$. For a given $n$ we have

$$
\begin{aligned}
D_{n} & =D\left(P_{Y^{n} \mathbf{X}^{n}} \| P_{Y^{n}} \times P_{\mathbf{X}^{n}}\right) \\
& \stackrel{(a)}{\geq} D\left(P_{Y^{n}} \hat{\mathbf{X}} \| P_{Y^{n}} \times P_{\hat{\mathbf{X}}^{n}}\right) \\
& \stackrel{(b)}{\geq} P_{H_{0}}\left(\mathcal{A}_{n}\right) \log \frac{P_{H_{0}}\left(\mathcal{A}_{n}\right)}{P_{H_{1}}\left(\mathcal{A}_{n}\right)}+P_{H_{0}}\left(\mathcal{A}_{n}^{c}\right) \log \frac{P_{H_{0}}\left(\mathcal{A}_{n}^{c}\right)}{P_{H_{1}}\left(\mathcal{A}_{n}^{c}\right)} \\
& \geq-\left(1-\alpha_{n}\right) \log \beta_{n}-h_{2}\left(\alpha_{n}\right)
\end{aligned}
$$

where $h_{2}(\cdot)$ is the binary entropy function. $(a)$ follows due to the data-processing inequality. (b) holds due to the log-sum inequality. Note that actually we have

$$
\begin{aligned}
D_{n} & =I_{P_{H_{0}}}\left(Y^{n} ; \mathbf{X}^{n}\right) \\
& =n H(Y)-H\left(Y^{n} \mid \mathbf{X}^{n}\right) \\
& \stackrel{(c)}{=} n H(Y)-H\left(Y^{n} \mid W, \mathbf{X}^{n}\right)-H(W) \\
& \quad+H\left(W \mid Y^{n}, \mathbf{X}^{n}\right) \\
& =n I(Y ; X)-\log M+H\left(W \mid Y^{n}, \mathbf{X}^{n}\right) .
\end{aligned}
$$

In $(c) W$ is independent of the users' sequences. Define the deterministic mapping $f_{n}: \mathcal{Y}^{n} \times \mathcal{X}^{n M} \rightarrow \mathcal{W} \cup\{e\}$ as follows. If there exists a unique index $\hat{w}$ such that $\left(y^{n}, x^{n}(\hat{w})\right) \in \mathcal{T}_{\epsilon}^{n}$ then we set $f_{n}\left(y^{n},(x(i))_{i=1}^{M}\right)$ to $\hat{w}$. If there is more than one or there is none then the corresponding value of $f_{n}$ is $e$. Then as $R<I(Y ; X)$ we have [1]

$$
\operatorname{Pr}_{H_{0}}\{W \neq \hat{W}\} \rightarrow 0, \text { as } n \rightarrow \infty .
$$

Therefore, by Fano's inequality we obtain

$$
H\left(W \mid Y^{n}, \mathbf{X}^{n}\right) \leq n \epsilon_{n},
$$

where $\epsilon_{n} \rightarrow 0$ as $n \rightarrow \infty$. Hence combining both expressions (18) and (19), the following bound holds for all sufficiently large $n$

$$
-\left(1-\alpha_{n}\right) \log \beta_{n}-h_{2}\left(\alpha_{n}\right) \leq n I(X ; Y)-\log M+n \epsilon_{n} .
$$

Hence,

$$
E \leq \liminf _{n \rightarrow \infty} \frac{1}{n} \log \frac{1}{\beta_{n}} \leq I(X ; Y)-R .
$$

The proof of Proposition 2 is complete.

Remark 2. Note that in the case of no compression our setting is an instance of hypothesis testing for the mixed source problem. When $M$ is a constant, we obtain a similar result as in [9, Example 4.1.1]. Therefore, Proposition 1 states that allowing the mixing coefficient to depend on $n$ can lead to a non-trivial reduction in the error exponent.

Another scenario where the lower bound is tight is given in the following proposition which is a slight generalization of the Ahlswede-Csiszar result on testing against independence [7, Theorem 2].

Proposition 2. Assume that $R=0$, i.e., the number of users in the database grows sub-exponentially, then we obtain

$$
E^{\star}\left(0, R_{c}\right)=\theta\left(0, R_{c}\right)=R_{\max }\left(R_{c}\right) .
$$

Proof. Similarly as in the proof of Proposition 2, we use the log-sum inequality to obtain the following expression

$D\left(P_{Y^{n} \phi_{n}\left(\mathbf{X}^{n}\right)} \| P_{Y^{n}} \times P_{\phi_{n}\left(\mathbf{X}^{n}\right)}\right) \geq-\left(1-\alpha_{n}\right) \log \beta_{n}-h_{2}\left(\alpha_{n}\right)$.

Using the data-processing inequality we obtain that

$$
\begin{aligned}
D\left(P_{Y^{n} \phi_{n}\left(\mathbf{X}^{n}\right) W} \| P_{Y^{n}} \times P_{\phi_{n}}\left(\mathbf{X}^{n}\right) \times P_{W}\right) \\
\geq-\left(1-\alpha_{n}\right) \log \beta_{n}-h_{2}\left(\alpha_{n}\right) .
\end{aligned}
$$

The left-hand side is equal to

$$
\begin{aligned}
H\left(Y^{n}\right) & -H\left(Y^{n} \mid \phi_{n}\left(\mathbf{X}^{n}\right), W\right)=n H(Y)-H\left(Y^{n} \mid J_{W}, W\right) \\
& =n\left(H(Y)-H\left(Y_{Q} \mid U\right)\right)
\end{aligned}
$$

where we first define $U_{i}=\left(J_{W}, Y^{i-1}\right)$ and then $U=\left(U_{Q}, Q\right)$ in which $Q$ is a uniform random variable on $[1: n]$ which is independent of everything else. For each $\delta>0$ the compression rate can be readily bounded (for all sufficiently large $n$ ) as

$$
R_{c}+\delta>I\left(X_{Q} ; U\right)
$$

Using the standard cardinality bounding technique [8, Appendix C] we can find an "equivalent" $\bar{U}$ and a probability kernel $P_{\bar{X} \mid \bar{U}}$ such that $|\overline{\mathcal{U}}| \leq|\mathcal{X}|+1, H\left(Y_{Q} \mid U\right)=H(\bar{Y} \mid \bar{U})$ and $H\left(X_{Q} \mid U\right)=H(\bar{X} \mid \bar{U})$ where $P_{\bar{X} \bar{Y}}=P_{X Y}$. Hence

$$
-\left(1-\alpha_{n}\right) \log \beta_{n}-h_{2}\left(\alpha_{n}\right) \leq n R_{\max }\left(R_{c}+\delta\right) .
$$

Taking $n \rightarrow \infty$ we obtain that

$$
E \leq R_{\max }\left(R_{c}+\delta\right), \forall \delta>0 .
$$

Taking $\delta \rightarrow 0$ we obtain that $E \leq \theta\left(0, R_{c}\right)$ for $R=0$.

Remark 3. Note that a central theme for the two aforementioned cases is that

$$
H\left(W \mid Y^{n},\left(J_{i}\right)_{i=1}^{M}\right) \approx n \epsilon_{n} .
$$

In the first case, this holds due to the fact that we can construct a decoding function by the identification problem. In the second case, the approximation is valid since the alphabet $\mathcal{W}$ has the sub-exponential size. If we restrict the compression mappings to the following set

$$
\begin{aligned}
& \mathcal{B}\left(R, R_{c}\right) \\
& =\left\{\left(\phi_{n}\right) \mid \text { there exists a sequence of }\left(\psi_{n}\right)\right. \text { such that }
\end{aligned}
$$




$$
\begin{gathered}
\limsup _{n \rightarrow \infty} \frac{1}{n} \log \left|\phi_{n}\right| \leq R_{c}, \\
\left.\lim _{n \rightarrow \infty} \operatorname{Pr}_{H_{0}}\left\{\psi_{n}\left(Y^{n}, \phi_{n}\left(\mathbf{X}^{n}\right)\right) \neq W\right\}=0\right\},
\end{gathered}
$$

then by following essentially the same steps as in the proof of Proposition 1, we can show that the restricted error exponent is characterized by $E_{\mathcal{B}}^{\star}\left(R, R_{c}\right)=\theta\left(R, R_{c}\right)$ when $R<R_{\max }\left(R_{c}\right)$.

\section{A Characterization via Strong Converse}

We assume in this section that $P_{X Y}(x, y)>0$ for all $(x, y) \in \mathcal{X} \times \mathcal{Y}$. Applying the blowing up approach from [7] is challenging, since both distributions are non-iid. We instead employ the information spectrum approach on top of the result by Ahlswede and Csiszar to show a partial converse. Similarly, we have the following definition.

Definition 3. Let $\epsilon \in[0,1)$ be an arbitrarily given constant. An error exponent $E$ of type II is $\epsilon$-achievable given $\left(R, R_{c}\right)$ if there exist enrollment and decision mappings $\left(\phi_{n}, \psi_{n}\right)$ such that

$$
\begin{gathered}
\limsup _{n \rightarrow \infty} \alpha_{n} \leq \epsilon, \limsup _{n \rightarrow \infty} \frac{1}{n} \log \left|\mathcal{M}_{1}\right| \leq R_{c}, \\
\liminf _{n \rightarrow \infty} \frac{1}{n} \log \frac{1}{\beta_{n}} \geq E .
\end{gathered}
$$

We define

$$
E_{\epsilon}^{\star}\left(R, R_{c}\right)=\sup \left\{E \mid E \text { is } \epsilon \text {-achievable given }\left(R, R_{c}\right)\right\} .
$$

We first present a lemma that relates our multi-user setting to the single-user setting of Ahlswede and Csiszar in [7].

Lemma 1. For any compression sequence $\left(\phi_{n}\right)$ and $\hat{E}$ we have

$$
\begin{aligned}
\operatorname{Pr}\{ & \left.\frac{1}{n} \log \frac{P_{Y^{n} \phi_{n}\left(\mathbf{X}^{n}\right)}\left(Y^{n}, \phi_{n}\left(\mathbf{X}^{n}\right)\right)}{P_{Y^{n}} \times P_{\phi_{n}\left(\mathbf{X}^{n}\right)}\left(Y^{n}, \phi_{n}\left(\mathbf{X}^{n}\right)\right)}>\hat{E}\right\} \\
\leq \operatorname{Pr} & \left\{\frac{1}{n} \log \frac{P_{\bar{Y}^{n} \phi_{n}\left(\bar{X}^{n}\right)}\left(\bar{Y}^{n}, \phi_{n}\left(\bar{X}^{n}\right)\right)}{P_{\bar{Y}^{n} \times P_{\phi_{n}\left(\bar{X}^{n}\right)}}\left(\bar{Y}^{n}, \phi_{n}\left(\bar{X}^{n}\right)\right)}>\hat{E}+\frac{1}{n} \log \frac{M}{2}\right\} \\
& +\frac{2 M}{e^{n \hat{E}}},
\end{aligned}
$$

where $\left(\bar{Y}^{n}, \bar{X}^{n}\right) \sim P_{Y X}^{\otimes n}$. Additionally, the left-hand side of (32) is evaluated with $\left(Y^{n}, \mathbf{X}^{n}\right) \sim P_{H_{0}}$ given in (1).

The first term in the RHS of (32) can be interpreted as the influence of the chosen user under hypothesis $H_{0}$, while the second ones reflects the interference of the other users under $H_{0}$.

Proof. For notation brevity we denote the LHS of (32) by $L_{n}$. Then we have

$$
\begin{aligned}
& L_{n}=\sum_{i=1}^{M} \frac{1}{M}
\end{aligned}
$$

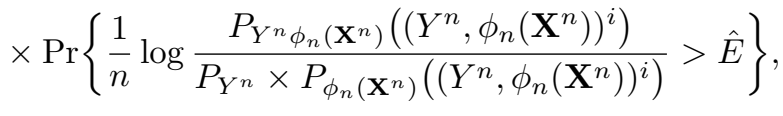

where $\left(Y^{n}, \phi_{n}\left(\mathbf{X}^{n}\right)\right)^{i} \sim P_{Y^{n} \phi_{n}\left(X^{n}(i)\right)} \times \prod_{j \neq i} P_{\phi_{n}\left(X^{n}(j)\right)}$ since under $H_{0}$ which is used to evaluate $L_{n}$ the following relation holds

$$
P_{Y^{n} \phi_{n}\left(\mathbf{X}^{n}\right)}\left(y^{n}, \phi_{n}\left(\mathbf{x}^{n}\right)\right)=\frac{1}{M} \sum_{k} P_{\left(Y^{n} \phi_{n}\left(\mathbf{X}^{n}\right)\right)^{k}}\left(y^{n}, \phi_{n}\left(\mathbf{x}^{n}\right)\right) .
$$

We further have the following bound for each $i \in[1: M]$ where $\gamma=e^{n \hat{E}}$

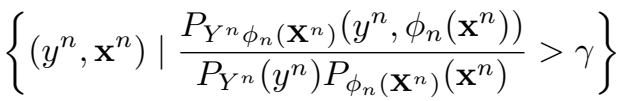

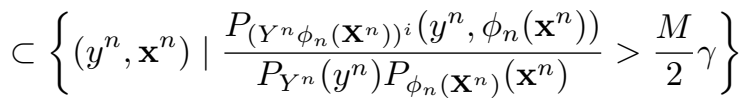

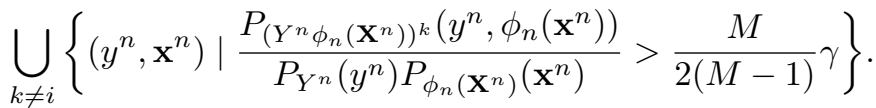

This leads to (34) shown on top of the next page. We have put more weight, $\frac{M}{2} \gamma$, in (33) to the corresponding $i$-th term to emphasize the influence of the chosen user. For each $k \neq i$ we have

$$
\begin{aligned}
t_{i k}= & \sum_{\left(y^{n}, x^{n}(i)\right)} P_{Y^{n}}\left(y^{n}\right) P_{\phi_{n}\left(X^{n}\right)}\left(\phi_{n}\left(x^{n}(i)\right)\right) \\
& \times \mathbf{1}\left\{\frac{P_{Y^{n} \phi_{n}\left(X^{n}\right)}\left(y^{n}, \phi_{n}\left(x^{n}(i)\right)\right)}{P_{Y^{n}}\left(y^{n}\right) P_{\phi_{n}\left(X^{n}\right)}\left(\phi_{n}\left(x^{n}(i)\right)\right)}>\frac{M}{2(M-1)} \gamma\right\} \\
& \leq \frac{2(M-1)}{M e^{n \hat{E}}} .
\end{aligned}
$$

The conclusion hence follows.

Roughly speaking, to provide a strong converse statement we aim to drive $L_{n}$ to 0 as $n \rightarrow \infty$. It can be seen that if $\hat{E}$ is greater than the spectral-sup mutual information of $\left(Y^{n}, \phi_{n}\left(\mathbf{X}^{n}\right)\right)$ then $L_{n}$ goes to 0 . However, the bound is hard to characterize in a single letter form. The following corollary shows that there exists a sequence $\left(L_{n_{k}}\right)$ which goes to 0 provided that some constraints are met. It will be shown later that the conclusion is sufficient for proving a partial strong converse statement.

Corollary 1. Given a compression sequence $\left(\phi_{n}\right)$ such that $\limsup _{n \rightarrow \infty} \frac{1}{n} \log \left|\mathcal{M}_{1}\right| \leq R_{c}$. If $\hat{E}=R_{\max }-R+2 \gamma$ where $R<R_{\max } / 2$ and $\gamma>0$ is arbitrary, then the exists a subsequence $\left(n_{k}\right)_{k=1}^{\infty}$ such that

$$
\lim _{k \rightarrow \infty} L_{n_{k}}=0 .
$$

Proof. The condition $R<R_{\max } / 2$ ensures that the second term in the RHS of (32) goes to 0 as $n \rightarrow \infty$. It suffices to show that there exists a subsequence $\left(n_{k}\right)$ such that the first term in the RHS of (32) converges to 0.

Define the following acceptance region for the single-user hypothesis testing problem

$\overline{\mathcal{A}}_{n}=\left\{\left(y^{n}, \phi_{n}\left(x^{n}\right)\right) \mid \frac{1}{n} \log \frac{P_{\bar{Y}^{n}} \phi_{n}\left(\bar{X}^{n}\right)}{P_{\bar{Y}^{n}} \times P_{\phi_{n}\left(\bar{X}^{n}\right)}}\left(y^{n}, \phi_{n}\left(x^{n}\right)\right)>\tilde{E}\right\}$, 


$$
\begin{aligned}
L_{n} & \leq \frac{1}{M} \sum_{i=1}^{M} \operatorname{Pr}\left\{\frac{1}{n} \log \frac{P_{\left(Y^{n} \phi_{n}\left(\mathbf{X}^{n}\right)\right)^{i}}}{P_{Y^{n}} \times P_{\phi_{n}\left(\mathbf{X}^{n}\right)}}\left(\left(Y^{n}, \phi_{n}\left(\mathbf{X}^{n}\right)\right)^{i}\right)>\hat{E}+\frac{1}{n} \log \frac{M}{2}\right\} \\
& +\frac{1}{M} \sum_{i=1}^{M} \sum_{k \neq i} \underbrace{\operatorname{Pr}\left\{\frac{P_{\left(Y^{n} \phi_{n}\left(\mathbf{X}^{n}\right)\right)^{i}}}{P_{Y^{n}} \times P_{\phi_{n}}\left(\mathbf{X}^{n}\right)}\left(\left(Y^{n}, \phi_{n}\left(\mathbf{X}^{n}\right)\right)^{k}\right)>\frac{M}{2(M-1)} \gamma\right\}}_{t_{i k}} \\
& =\operatorname{Pr}\left\{\frac{1}{n} \log \frac{P_{\bar{Y}^{n} \phi_{n}\left(\bar{X}^{n}\right)}\left(\bar{Y}^{n}, \phi_{n}\left(\bar{X}^{n}\right)\right)}{P_{\bar{Y}^{n}} \times P_{\phi_{n}\left(\bar{X}^{n}\right)}\left(\bar{Y}^{n}, \phi_{n}\left(\bar{X}^{n}\right)\right)}>\hat{E}+\frac{1}{n} \log \frac{M}{2}\right\}+\frac{1}{M} \sum_{i=1}^{M} \sum_{k \neq i} t_{i k} .
\end{aligned}
$$

where $\tilde{E}=R_{\max }+\gamma$. Then it can be seen that

$$
\begin{aligned}
\bar{\beta}_{n} & =P_{\bar{Y}^{n}} \times P_{\phi_{n}\left(\bar{X}^{n}\right)}\left(\overline{\mathcal{A}}_{n}\right) \\
& =\sum_{\left(y^{n}, \phi_{n}\left(x^{n}\right)\right) \in \overline{\mathcal{A}}_{n}} P_{\bar{Y}^{n}}\left(y^{n}\right) P_{\phi_{n}\left(\bar{X}^{n}\right)}\left(\phi_{n}\left(x^{n}\right)\right) \\
& \leq e^{-n \tilde{E}} \sum_{\left(y^{n}, \phi_{n}\left(x^{n}\right)\right) \in \overline{\mathcal{A}}_{n}} P_{\bar{Y}^{n} \phi_{n}\left(\bar{X}^{n}\right)}\left(y^{n}, \phi_{n}\left(x^{n}\right)\right) \\
& \leq e^{-n \tilde{E}}
\end{aligned}
$$

or $\liminf _{n \rightarrow \infty} \frac{1}{n} \log \frac{1}{\beta_{n}} \geq \tilde{E}$. Since $\tilde{E}>R_{\max }$ and $P_{X Y}(x, y)>0$ for all $(x, y)$, by the strong converse result of Ahlswede and Csiszar [7, Theorem 3] we have

$$
\limsup _{n \rightarrow \infty} \bar{\alpha}_{n}=1 \text {, where } \bar{\alpha}_{n}=P_{\bar{Y}^{n} \phi_{n}\left(\bar{X}^{n}\right)}\left(\overline{\mathcal{A}}_{n}^{c}\right) \text {. }
$$

Then there exists a subsequence $\left(n_{k}\right)_{k=1}^{\infty}$ such that

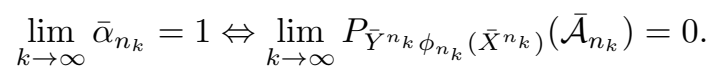

Additionally, for all sufficiently large $n$ we have

$$
\hat{E}+\frac{1}{n} \log \frac{M}{2}>\tilde{E}
$$

By Lemma 1 and (39) we obtain then

$$
\lim _{k \rightarrow \infty} L_{n_{k}}=0
$$

We now summarize the above analysis in the following theorem which is a partial strong converse statement.

Theorem 2. The strong converse holds when $R<R_{\max } / 2$, i.e., $\forall \epsilon, 0 \leq \epsilon<1$,

$$
E_{\epsilon}^{\star}\left(R, R_{c}\right)=\theta\left(R, R_{c}\right)=R_{\max }-R, \text { if } R<R_{\max } / 2 .
$$

It is interesting to note that Theorem (2) shows the tightness of $E^{\star}\left(R, R_{c}\right)$ for $R<R_{\max } / 2$. It is not clear whether the same statement can be reached with the methods in Section II.

Proof. Suppose that there exists a sequence of compression mappings $\left(\phi_{n}\right)$ and a sequence of decision mappings $\left(\psi_{n}\right)$ such that

$$
\begin{aligned}
\limsup _{n \rightarrow \infty} \alpha_{n} \leq \epsilon, \limsup _{n \rightarrow \infty} \frac{1}{n} \log \left|\mathcal{M}_{1}\right| \leq R_{c}, \\
\\
\text { and } \liminf _{n \rightarrow \infty} \frac{1}{n} \log \frac{1}{\beta_{n}} \geq E .
\end{aligned}
$$

Then given $\gamma>0$ small enough such that $\epsilon+\gamma<1$ and $E-\gamma>0$ for sufficiently all large $n$ we have $\alpha_{n} \leq \epsilon+\gamma$ and $\beta_{n} \leq e^{-n(E-\gamma)}$. Let $\hat{E}=R_{\max }-R+2 \gamma$ and $\left(n_{k}\right)$ be the corresponding subsequence such that (36) holds. Then as [9, Lemma 4.1.2], c.f. also [10, Section 13.1],

$$
\begin{aligned}
1-\alpha_{n} & -e^{n \hat{E}} \beta_{n} \\
& \leq \operatorname{Pr}\left\{\frac{1}{n} \log \frac{P_{Y^{n} \phi_{n}\left(\mathbf{X}^{n}\right)}}{P_{Y^{n}} \times P_{\phi_{n}}\left(\mathbf{X}^{n}\right)}\left(Y^{n}, \phi_{n}\left(\mathbf{X}^{n}\right)\right)>\hat{E}\right\},
\end{aligned}
$$

holds for any $n$, where the RHS is actually $L_{n}$, we obtain for all sufficiently large $n_{k}$

$$
\begin{aligned}
1-\epsilon-\gamma-e^{n_{k} \hat{E}} e^{-n_{k}(E-\gamma)} & \leq L_{n_{k}} \\
\Longrightarrow \hat{E}-E+\gamma & \geq \frac{1}{n_{k}} \log \left(1-\epsilon-\gamma-L_{n_{k}}\right) \\
\Longrightarrow R_{\max }-R+3 \gamma & \geq E \stackrel{\gamma \rightarrow 0}{\Longrightarrow} R_{\max }-R \geq E \\
\Longrightarrow R_{\max }-R & \geq E_{\epsilon}^{\star}\left(R, R_{c}\right) .
\end{aligned}
$$

\section{REFERENCES}

[1] F. M. J. Willems, T. Kalker, and J.-P. Linnartz, "On the capacity of a biometrical identification system," in International Symposium on Information Theory, 2003.

[2] E. Tuncel, "Capacity/storage tradeoff in high-dimensional identification systems," IEEE Trans. Inf. Theory, vol. 55, no. 5, pp. 2097-2106, 2009.

[3] E. Tuncel and D. Gündüz, "Identification and lossy reconstruction in noisy databases," IEEE Trans. Inf. Theory, vol. 60, no. 2, pp. 822-831, 2014.

[4] S. Voloshynovskiy, O. Koval, F. Beekhof, F. Farhadzadeh, and T. Holotyak, "Information-theoretical analysis of private content identification," in IEEE Information Theory Workshop (ITW). IEEE, 2010, pp. $1-5$.

[5] N. A. Schmid, "Large deviations performance analysis for biometrics recognition," in 40th Annual Allerton Conference on Communication, Control, and Computing (Allerton), 2002.

[6] P. Moulin, "Statistical modeling and analysis of content identification," in Information Theory and Applications Workshop (ITA). IEEE, 2010, pp. $1-5$.

[7] R. Ahlswede and I. Csiszár, "Hypothesis testing with communication constraints," IEEE Trans. Inf. Theory, vol. 32, no. 4, pp. 533-542, 1986.

[8] A. El Gamal and Y.-H. Kim, Network information theory. Cambridge university press, 2011.

[9] T. S. Han, Information-Spectrum Methods in Information Theory. Springer-Verlag Berlin Heidelberg, 2003.

[10] Y. Polyanskiy and Y. Wu, "Lecture notes on information theory," MIT (6.441), UIUC (ECE 563), 2017. 